\title{
Revisando un órgano olvidado: Evaluación del timo en PET-CT
}

\section{Revisiting a Forgotten Organ: Thymus Evaluation in PET-CT}

\author{
Daniel Hasson ${ }^{1}$ Giancarlo Schiappacasse ${ }^{2}$ Julia Alegría ${ }^{2}$ Claudio Silva $^{2}$
}

\author{
${ }^{1}$ Residente de Radiología, Facultad de Medicina Clínica Alemana \\ Universidad del Desarrollo, Vitacura, Santiago, Chile \\ 2 Radiólogo, Facultad de Medicina Clínica Alemana Universidad del \\ Desarrollo, Vitacura, Santiago, Chile
}

Address for correspondence Daniel Hasson, MD, Facultad de Medicina Clínica Alemana Universidad del Desarrollo, Vitacura, Santiago, Chile (e-mail: dhasson@alemana.cl).

Rev Argent Radiol 2020;84:55-60.

\section{Resumen \\ Palabras Clave \\ - timo \\ - neoplasia tímica \\ - tomografía computada por emisión de positrones}

La pesquisa incidental de lesiones tímicas ha aumentado. Una adecuada aproximación a esas lesiones en la tomografía computada por emisión de positrones (PET-CT) es fundamental, pues se usa como parte de la mayoría de los procedimientos de planificación oncológica. Se han seleccionado casos representativos respecto de los aspectos más importantes de las imágenes de timo en PET-CT y cómo esa técnica puede contribuir a un diagnóstico preciso o a la planificación del tratamiento. Específicamente, presentamos una descripción general de las lesiones tímicas comunes y los imitadores de enfermedad, con énfasis en los hallazgos en PET-CT, incorporando también ejemplos de resonancia magnética (RM).

Incidental thymic lesion findings have increased. An adequate characterization of these lesions in positron emission computed tomography (PET-CT) is essential, since it is used as part of most oncological planning procedures. Representative cases have been selected regarding the most important aspects of thymus imaging in PET-CT and how this technique can contribute to an accurate diagnosis or treatment planning. Specifically, we present a general description of common thymic lesions and disease mimics, with an emphasis on PET-CT findings, also incorporating examples of magnetic resonance imaging.

\section{Introducción}

El timo es un órgano linfático que desempeña un rol fundamental en el desarrollo y maduración del sistema inmune durante la infancia, específicamente las células T. ${ }^{1}$ Los tumores tímicos representan aproximadamente el 50\% de las lesiones del mediastino anterior, el resto corresponden en su mayoría a linfomas y teratomas. ${ }^{2}$

Aunque se puede lograr un diagnóstico definitivo de lesiones tímicas por medio de un estudio histológico, una caracterización y estadificación apropiadas puede impactar significativamente en su manejo. La tomografía computada por emisión de positrones con tomografía multicorte (PET-CT) con 18F-fluorodeoxiglucosa (18F-FDG) combina información funcional y anatómica, que determinan mayor precisión diagnóstica y en la determinación de la extensión de enfermedades neoplásicas. La resonancia magnética (RM), además de entregar una insuperable resolución espacial, es especialmente beneficiosa para identificar lesiones quísticas.

\section{received}

January 15, 2020

accepted

January 15, 2020
DOI https://doi.org/

10.1055/s-0040-1702992.

ISSN 1852-9992.
Copyright $\odot$ 2020, Sociedad Argentina de Radiología. Publicado por Thieme Revinter Publicações Ltda., Rio de Janeiro, Brazil. Todos los derechos reservados.

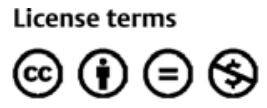




\section{Desarrollo}

Se presenta una revisión iconográfica de los principales hallazgos imagenológicos por 18F-FDG PET-CT del timo en situaciones fisiológicas y patológicas. Se acompañan con imágenes de RM en casos en que se estimó pertinente. Se agruparon las diferentes entidades como:

- Variación con la edad

- Hiperplasia tímica

- Quistes tímicos

- Tumores epiteliales tímicos

- Linfoma tímico

\section{Variación con la edad}

El timo es un órgano linfático que desempeña un rol fundamental en el desarrollo del sistema inmunológico durante la infancia, y en consonancia con eso, experimenta cambios dinámicos con la edad y la enfermedad. ${ }^{1}$ En pacientes jóvenes, puede mostrar predominantemente atenuación de tejidos blandos, mientras que, en pacientes mayores, puede presentar reemplazo adiposo completo.

Aunque existen casos esporádicos de captación tímica fisiológica en adultos normales en los estudios con 18FFDG PET-CT, la captación tímica es más notoria antes de la pubertad, que es el punto en el que el timo sufre infiltración grasa e involución. 2,3

Normalmente, la captación tímica fisiológica es de baja intensidad. Los valores máximos de captación (SUVmáx) del timo normal se han situado entre aproximadamente $1,0 \mathrm{y}$ 1,8 , aunque existe un cierto grado de traslape entre esos valores y los que se encuentran en la hiperplasia tímica (-Fig. 1). ${ }^{4}$

\section{Hiperplasia tímica}

Existen dos tipos histológicos distintos de hiperplasia tímica: hiperplasia tímica verdadera e hiperplasia folicular linfoide. La naturaleza del aumento de tamaño del timo difiere en ambos tipos de hiperplasia: en la hiperplasia tímica verdadera, aumenta la masa de tejido tímico, preservando la arquitectura histológica. En la hiperplasia folicular linfoide, el aumento de volumen de los centros germinales linfoides constituye el aumento del tamaño de la glándula. La hiperplasia tímica verdadera o hiperplasia de rebote es un fenómeno caracterizado por un aumento de tamaño del timo posterior a un factor estresante, como la quimioterapia, radiación, tratamiento con cortico-esteroides, quemaduras o cirugía. ${ }^{5}$ La hiperplasia folicular linfoide se asocia a numerosos trastornos crónicos inflamatorios y autoinmunes, principalmente miastenia gravis, como también a la enfermedad de Graves, lupus eritematoso sistémico, artritis reumatoide $\mathrm{y}$ otras afecciones autoinmunes. ${ }^{6}$

Aunque los dos tipos de hiperplasia tímica son imposibles de diferenciar mediante imágenes, la hiperplasia tímica generalmente puede distinguirse de procesos expansivos en base a ciertos hallazgos orientadores. La hiperplasia tímica generalmente se presenta como un aumento difuso y simétrico de volumen tímico, de contornos suaves, con densidad intercalada de grasa y de partes blandas,

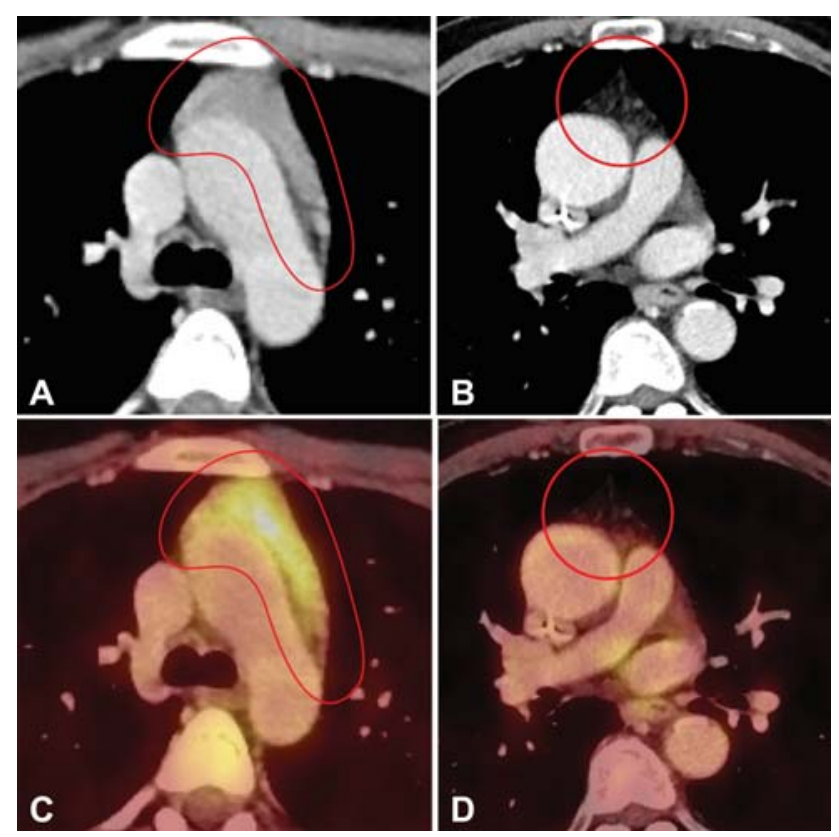

Fig. 1 Timo fisiológico: variación con la edad. Imágenes axiales. TC con contraste intravenoso (IV) (A y B) y fusión PET-TC (Cy D). Pacientes de 20 y 65 años (izquierda y derecha respectivamente) sin antecedentes de quimioterapia. La paciente de 20 años presenta un timo cuadrilateral, con escasa captación de 18-FDG, mientras que el paciente de 65 años presenta un timo triangular sin captación significativa de 18-FDG, demostrando la degeneración grasa propia del proceso involutivo asociado al envejecimiento.

manteniendo los planos grasos de clivaje (-Fig. 2). En contraste con lo anterior, un tumor tímico puede demostrar una masa focal, contornos nodulares, heterogeneidad (que representa hemorragia o necrosis) y/o calcificaciones. ${ }^{7}$ Los valores SUVmáx de 18F-FDG en hiperplasia tímica se sitúan aproximadamente entre 2,0 y $2,8 .^{2}$ Se puede emplear la imagen de RM por desplazamiento químico (chemical shift). Así, en la hiperplasia tímica, podemos observar una caída de señal en las imágenes fuera de fase respecto de las imágenes en fase, debido a la presencia de grasa microscópica en el timo hiperplásico (-Fig. 3). ${ }^{8}$

\section{Quistes tímicos}

Los quistes tímicos son lesiones relativamente infrecuentes, sin predominancia en algún grupo etario. Pueden ser de origen congénito o adquirido. Los quistes congénitos pueden encontrarse en cualquier ubicación a lo largo del conducto timofaríngeo, mientras que los adquiridos se desarrollan posterior a quimioterapia, toracotomías o en asociación con tumores tímicos. ${ }^{9}$ En imágenes tomográficas se presentan como formaciones nodulares, con densidad líquida, con paredes finas o imperceptibles; pueden presentar septos en su interior. En ocasiones, sus paredes pueden presentar un tenue realce con el contraste intravenoso. Su apariencia puede ser menos característica cuando se complican con infección o hemorragia, en las que pueden ser algo heterogéneos, lo que puede plantear un diagnóstico diferencial con nódulos sólidos. La RM con contraste puede ser de utilidad para distinguir fluido hemorrágico de tejido de partes blandas. ${ }^{10}$ En el examen PET-CT, esas lesiones no presentan captación de 18F-FDG (-Fig. 4). 


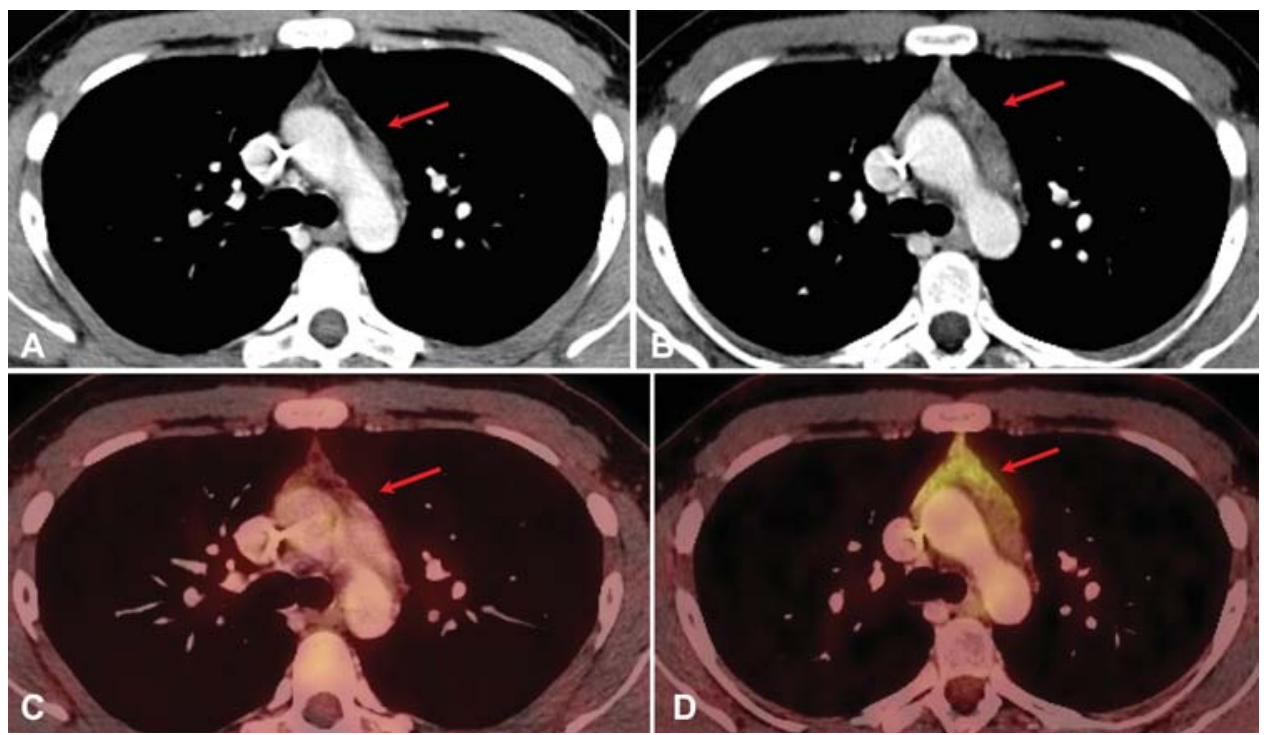

Fig. 2 Hiperplasia de rebote. Imágenes axiales. TC con contraste IV (Ay B) y fusión PET-TC (Cy D). Paciente varón de 21 años se sometió a quimioterapia por una neoplasia testicular. Imágenes pre $(A$ y $C$ ) y post (B y D quimioterapia revelan un leve aumento de tamaño del timo y significativo aumento de captación de 18-FDG (SUVmáx: 3,8). Se interpretó como hiperplasia de rebote. El paciente continúa sin cambios tras dos años de seguimiento.
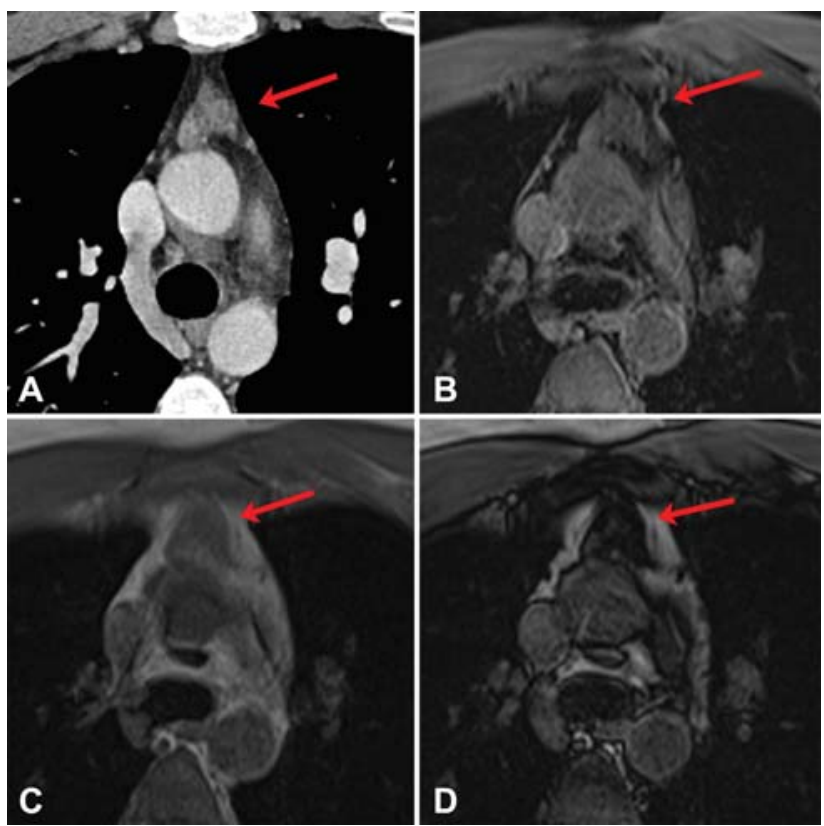

Fig. 3 Hiperplasia tímica. Imágenes axiales. En TC no contrastada (A) se reconoce formación con densidad de partes blandas de morfología nodular en el mediastino prevascular, con discreto realce con contraste paramagnético en RM (B). Esa lesión presenta caída de señal en la secuencia fuera de fase en las secuencias de eco gradiente (Cy D).

\section{Tumores epiteliales tímicos}

Los tumores epiteliales tímicos incluyen el timoma y el carcinoma tímico, constituyendo los tumores predominantes de origen tímico. La clasificación histológica de la Organización Mundial de la Salud (OMS) divide a los timomas en A, AB, B1, B2, $\mathrm{B} 3$, o C, de acuerdo a la morfología y grado de atipia de las células epiteliales tumorales en grados creciente de malignidad. ${ }^{11}$ Se han propuesto numerosas clasificaciones de estadificación para tumores epiteliales del timo, siendo la más usada la MasaokaKoga. ${ }^{12}$ Sin embargo, la recientemente propuesta clasificación
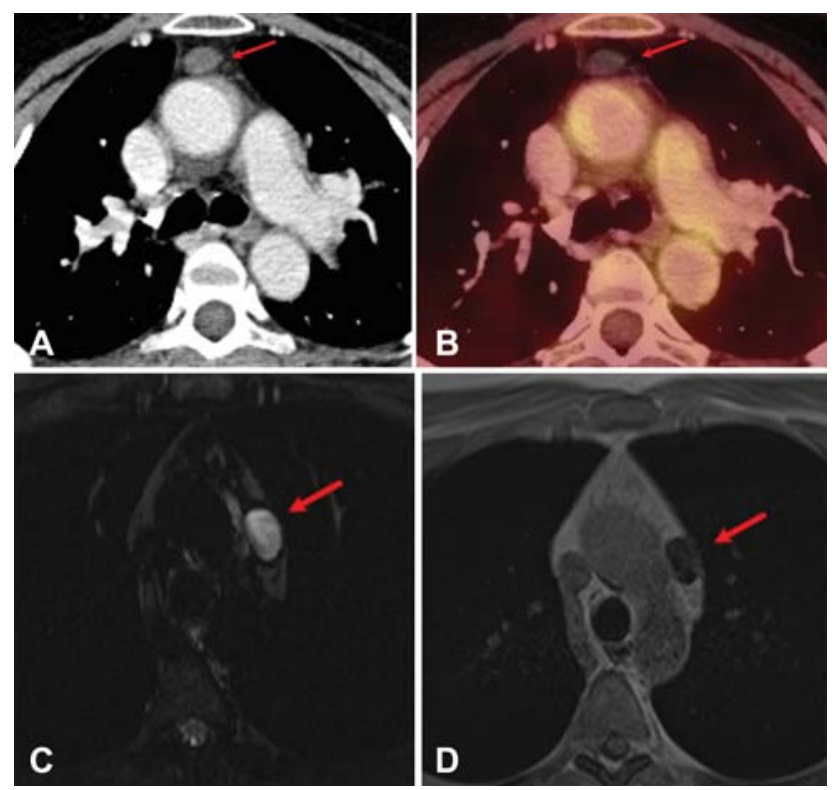

Fig. 4 Quiste tímico. Imágenes axiales TC con contraste IV y fusión PET-TC, (A y B) en las que se evidencia un nódulo bien delimitado con densidad líquida, de paredes imperceptibles, sin realce con contraste IV ni captación de 18-FDG. Imágenes axiales de RM de tórax de otro caso muestran una formación nodular hiperintensa en secuencias ponderadas en $\mathrm{T} 2$ (C) e hipointensa en secuencias ponderadas en T1 (D), con un plano de clivaje graso con la pared lateral izquierda del arco aórtico, y que no deforma el contorno mediastínico.

International Association for the Study of Lung Cancer / International Thymic Malignancy Interest Group (IASLC/ ITMIG), parece muy promisoria por su robustez metodológica y su incorporación a la octava edición del manual de estadificación del American Joint Committee on Cancer (AJCC). ${ }^{13}$

El timoma es la masa mediastínica anterior más frecuente en adultos. ${ }^{7}$ Aunque generalmente son asintomáticos, de 20\% a 30\% de los pacientes pueden presentar síntomas de compresión torácica, miastenia gravis, aplasia pura de serie roja u otros 

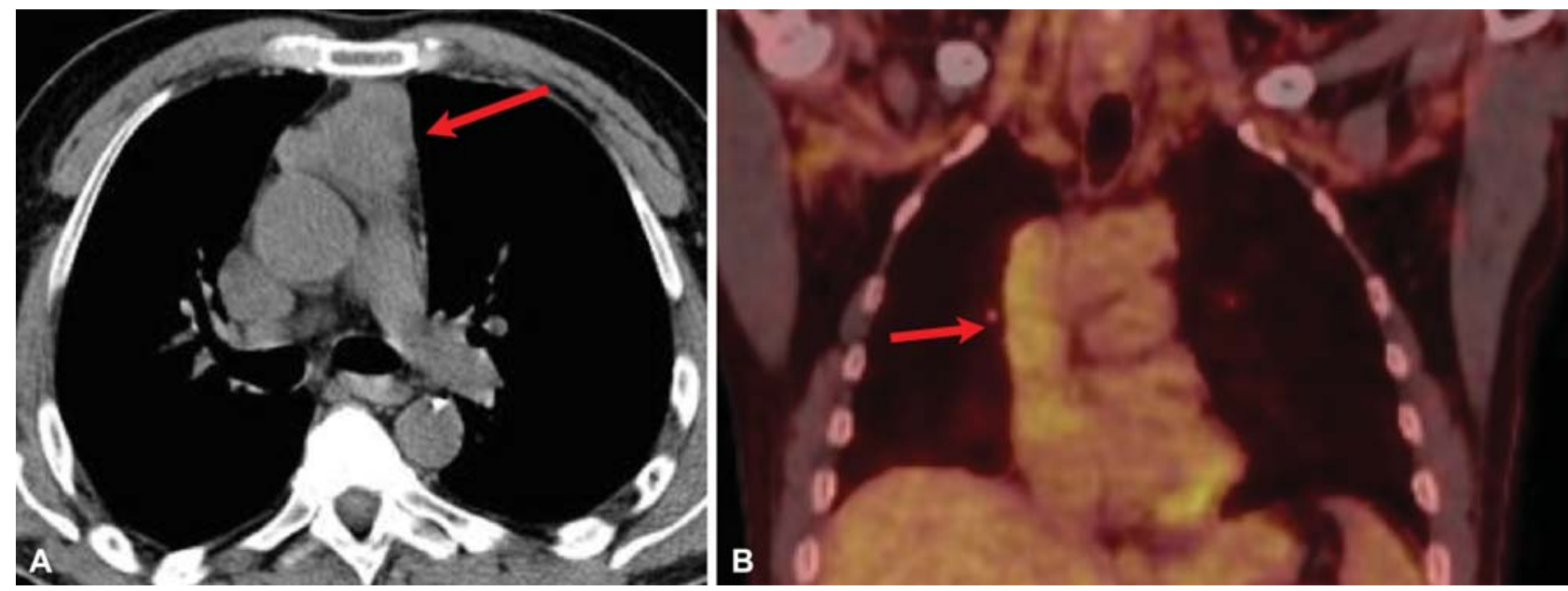

Fig. 5 Timoma invasivo. Imágenes axiales. Varón de 52 años. La TC no contrastada (A) demuestra una masa prevascular con pérdida de plano graso. En la fusión PET-TC, visión coronal (B) se reconoce una masa hipermetabólica (SUVmáx 4,5) que se extiende a través de la vena cava superior al atrio derecho.

trastornos inmunitarios. En particular, la relación entre timoma y miastenia gravis está claramente establecida: un $15 \%$ de los pacientes con miastenia gravis presentarán timoma. ${ }^{14}$

El rol de las imágenes en los tumores epiteliales tímicos es identificar y estadificar con precisión, poniendo énfasis en la detección de signos de enfermedad avanzada, como invasión local, adenopatías y diseminación a distancia. ${ }^{13} \mathrm{La}$ identificación de pacientes con enfermedad avanzada es muy relevante, ya que la quimioterapia neo-adyuvante preoperatoria puede administrarse con el fin de lograr una resección quirúrgica completa, mejorando el pronóstico de estos pacientes. ${ }^{15}$

En timomas, la imagen en TC generalmente evidencia una masa homogénea de partes blandas con bordes bien delimitados y una morfología lobulada, redonda u ovalada. En ocasiones, se pueden ver calcificaciones o componentes de baja densidad que representan necrosis (especialmente en tumores grandes) (- Fig. 5). ${ }^{16}$ Hasta el 5\% de los timomas invasivos pueden tener metástasis al momento de presentación y el PET-CT, siendo un método de evaluación de todo el cuerpo, es de utilidad para detectarlas. ${ }^{17}$

La capacidad de 18F-FDG PET-CT para diferenciar los timomas invasivos (alto riesgo) y no invasivos (bajo riesgo) suscita controversias, pues se observa una marcada superposición en la captación de timomas no invasivos e invasivos. Sin embargo, existe un relativo consenso en que los timomas invasivos presentan mayor captación en comparación con los no-invasivos. ${ }^{18}$

El carcinoma tímico puede ser difícil de distinguir mediante imágenes de un timoma localmente avanzado, pero el carcinoma tímico tiende a ser más agresivo, con rápida invasión de estructuras adyacentes. El carcinoma tímico por lo general carece de cápsula y las adenopatías y metástasis a distancia orientan a ese diagnóstico. Generalmente, se presentan en TC como masas multilobuladas, heterogéneas con áreas de necrosis y calcificación. Su captación es alta, típicamente con un SUVmáx mayor a 7 (-Fig. 6). ${ }^{6}$

Las metástasis a distancia son infrecuentes al momento de la presentación en tumores epiteliales tímicos. Su ubicación más común es en pleura (drop metastases), mientras que son menos frecuentes en riñones, hígado hueso y cerebro. ${ }^{19}$

\section{Linfoma}

El linfoma es la causa más frecuente de masa mediastínica prevascular en niños y adultos jóvenes. ${ }^{20}$ El compromiso tímico
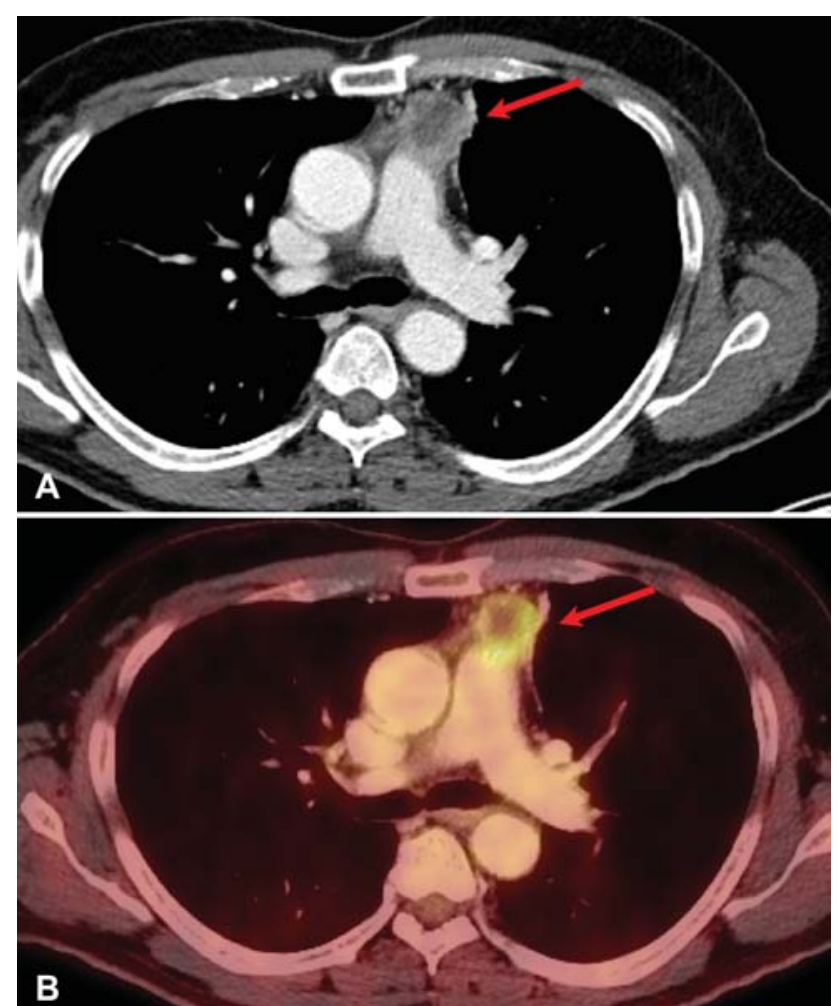

Fig. 6 Carcinoma tímico. Varón de 66 años. Masa polilobulada prevascular que en TC con contraste IV axial (A) demuestra componente sólido periférico que realza y un centro no realzante compatible con necrosis. No se identifica un plano de clivaje con la arteria pulmonar. En fusión PET-TC (B), presenta alta captación del radiotrazador en la porción sólida (SUVmáx: 17.5). 

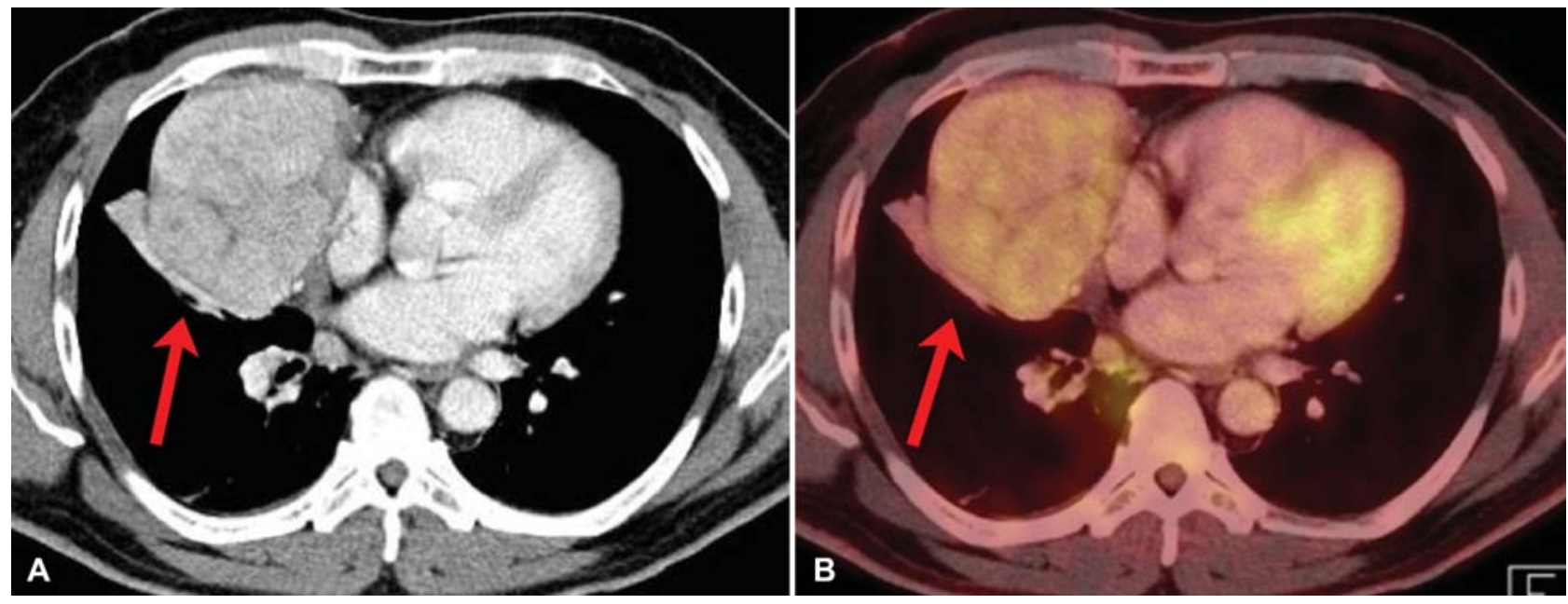

Fig. 7 Linfoma no-Hodgkin. Imágenes axiales. Masa prevascular con realce levemente heterogéneo en TC con contraste IV (A). En fusión PET-CT (B) se identifica discreto hipermetabolismo (SUVmáx 4.2).

en linfoma ocurre en general en el contexto de enfermedad diseminada, pero ocasionalmente puede verse compromiso aislado del timo. En las imágenes puede ser difícil de distinguir de otras masas tímicas. En TC, puede aparecer como aumento de volumen difuso, una masa dominante o múltiples masas separadas. ${ }^{13}$ Una apariencia más sugerente es la de un aumento de volumen tímico nodular y asimétrico. La presencia de una masa de esas características en un paciente joven asociado a adenopatías orienta al diagnóstico. En PET-CT se reconocen hallazgos similares, asociados a incrementos de SUVmáx, generalmente sobre 3,4 (-Fig. 7). Se considera que el valor de SUVmáx es predictivo de agresividad: los valores mayores a 13 sugieren linfomas agresivos y los menores a 6 , linfomas indolentes. ${ }^{21}$

\section{Confidencialidad de los Datos}

Los autores declaran que han seguido los protocolos de su centro de trabajo sobre la publicación de datos de pacientes y que todos los pacientes incluidos en el estudio han recibido información suficiente y han dado su consentimiento informado por escrito.

Conflicto de Intereses

Los autores declaran no tener ningún conflicto de intereses.

\section{Bibliografía}

1 Lee HM, Bautista JL, Hsieh CS. Thymic and peripheral differentiation of regulatory T cells. Adv Immunol. 2011;112:25-71

2 Liu Y. Characterization of thymic lesions with F-18 FDG PET-CT: an emphasis on epithelial tumors. Nucl Med Commun. 2011;32 (07):554-562

3 Wittram C, Fischman AJ, Mark E, Ko J, Shepard JA. Thymic enlargement and FDG uptake in three patients: CT and FDG positron emission tomography correlated with pathology. AJR Am J Roentgenol. 2003;180(02):519-522

4 Brink I, Reinhardt MJ, Hoegerle S, Altehoefer C, Moser E, Nitzsche EU. Increased metabolic activity in the thymus gland studied with $18 \mathrm{~F}-$ FDG PET: age dependency and frequency after chemotherapy. J Nucl Med. 2001;42(04):591-595
5 Sharma P, Singhal A, Kumar A, Bal C, Malhotra A, Kumar R. Evaluation of thymic tumors with 18F-FDG PET-CT: a pictorial review. Acta Radiol. 2013;54(01):14-21

6 Goldstein AJ, Oliva I, Honarpisheh H, Rubinowitz A. A tour of the thymus: a review of thymic lesions with radiologic and pathologic correlation. Can Assoc Radiol J. 2015;66(01):5-15

7 Santana L, Givica A, Camacho C, Armed Forces Institute of Pathology. Best cases from the AFIP: thymoma. Radiographics. 2002;22(Spec No):S95-S102

8 Inaoka T, Takahashi K, Mineta M, Yamada T, Shuke N, Okizaki A, et al. Thymic hyperplasia and thymus gland tumors: differentiation with chemical shift MR imaging. Radiology. 2007;243(03):869-876

9 Choi YW, McAdams HP, Jeon SC, Hong EK, Kim YH, Im JG, et al. Idiopathic multilocular thymic cyst: CT features with clinical and histopathologic correlation. AJR Am J Roentgenol. 2001;177(04): 881-885

10 Nishino M, Ashiku SK, Kocher ON, Thurer RL, Boiselle PM, Hatabu H. The thymus: a comprehensive review. Radiographics. 2006;26 (02):335-348

11 Travis WD, Brambilla E, Burke AP, Marx A, Nicholson AG. Introduction to The 2015 World Health Organization Classification of Tumors of the Lung, Pleura, Thymus, and Heart. J Thorac Oncol. 2015;10(09):1240-1242

12 Detterbeck FC, Nicholson AG, Kondo K, Van Schil P, Moran C. The Masaoka-Koga stage classification for thymic malignancies: clarification and definition of terms. J Thorac Oncol. 2011;6(07, Suppl 3):S1710-S1716

13 Carter BW, Benveniste MF, Madan R, Godoy MC, de Groot PM, Truong MT, et al. ITMIG Classification of Mediastinal Compartments and Multidisciplinary Approach to Mediastinal Masses. Radiographics. 2017;37(02):413-436

14 Rosado-de-Christenson ML, Galobardes J, Moran CA. Thymoma: radiologic-pathologic correlation. Radiographics. 1992;12(01): 151-168

15 Ströbel P, Bauer A, Puppe B, Kraushaar T, Krein A, Toyka K, et al. Tumor recurrence and survival in patients treated for thymomas and thymic squamous cell carcinomas: a retrospective analysis. J Clin Oncol. 2004;22(08):1501-1509

16 McErlean A, Huang J, Zabor EC, Moskowitz CS, Ginsberg MS. Distinguishing benign thymic lesions from early-stage thymic malignancies on computed tomography. J Thorac Oncol. 2013;8 (07):967-973

17 Bott-Kothari T, Aron BS, Bejarano P. Malignant thymoma with metastases to the gastrointestinal tract and ovary: a case 
report and literature review. Am J Clin Oncol. 2000;23(02): $140-142$

18 Kitami A, Sano F, Ohashi S, Suzuki K, Uematsu S, Suzuki T, et al. The Usefulness of Positron-Emission Tomography Findings in the Management of Anterior Mediastinal Tumors. Ann Thorac Cardiovasc Surg. 2017;23(01):26-30

19 Thomas CR, Wright CD, Loehrer PJ. Thymoma: state of the art. J Clin Oncol. 1999;17(07):2280-2289
20 Juanpere S, Cañete N, Ortuño P, Martínez S, Sanchez G, Bernado L. A diagnostic approach to the mediastinal masses. Insights Imaging. 2013;4(01):29-52

21 Alobthani G, Romanov V, Isohashi K, Matsunaga K, Watabe T, Kato $\mathrm{H}$, et al. Value of ${ }^{18} \mathrm{~F}$-FDG PET/CT in discrimination between indolent and aggressive non-Hodgkin's lymphoma: A study of 328 patients. Hell J Nucl Med. 2018;21(01):7-14 